\title{
Particle-based Methodology for Representing Mobile Ad-Hoc Networks
}

\author{
Carlos J. Martinez ${ }^{1}{ }^{2}$, Marisa López ${ }^{2}$ IEEE member, Luis C. Estebanez ${ }^{1}$, \\ ${ }^{1}$. Hispatel Ing. SRL. \\ c/ La Glorieta, 52 \\ Churra , 30110 Murcia \\ SPAIN \{www.InterQuanta.com \} \\ ${ }^{2}$. Dpto. Ingeniería Electrónica \\ Ciudad Universitaria s/n - 28040 Madrid \\ SPAIN \{email: jcmartinez@die.upm.es \}
}

\begin{abstract}
We present a methodology based on physics laws and particles in order to represent, simulate, and architect advanced networking models. We introduce a mathematical formalism with its basic postulates seeing the messages signals and nodes as interacting/colliding particles and space-temporal zones, respectively. In particular we focus on using this "Particle" view for Ad-Hoc networks, where we can represent network nodes as the moving particles in physics. This idea can be used in several types of networks like sensor or mobile, and even to computational systems, this first presentation aims to continue with further work on robustness, tools, and examples on how this view can be used in specification of complex networks in conjunction with existing methodologies and tools.
\end{abstract}

Index Terms - methodology, model, physics, heterogeneous, ad-hoc networks, particles, tools, ubiquitous

\section{INTRODUCTION}

$\mathrm{T}$ HE constantly evolving network and mobility technology is also followed by new and complex ways to represent and work with these networks, from design to deployment. Given the enormous complexity of the new networking paradigms, it is important to help designers simplifying the process of representing models.

A key issue in today's wide band communication is time, which plays a more important role in mobile systems than in fixed infrastructures. In classical infrastructures, the message delivery is the primary concern but in mobile networks the time at which a message arrives can become more important since the destination might be not accessible an instant later, resulting in mobility-induced failures [5].

Other key characteristics are a good visual representation and having modeling methods with the ability to capture the

This work was supported in part by the private company Hispatel Ing Srl and InterQuanta.com 's research funds. The author worked in this research while at Hispatel Ing. Srl (during 2003-2004 based on initial works in year 2000 ) and is now consultant with organizations and companies not related to the subject of this paper. Manuscript received Jan 14, 2006 spatial information, critical in networks. Our methodology formalizes the spatial coordinates integrating them naturally in any model or system representation, the complexity of modern designs can thus reuse the classical visual tools while having a formal representation that we will introduce here.

In this paper we introduce a methodology to represent and implement networks based on physics, such as particles that collide and follow the physics dynamics laws. The modeling or methodology resulting from this idea could be named "particle dynamics," "collision," or "particle networks," since it relies on the collisions or reactions among particles. It does not require a great depth of particle-physics knowledge, and even the use of common physics like billiards-balls is perfectly suitable to ease up design efforts.

Among the wide variety of methodologies for modeling systems, we find that basing our representation in nature ended up in being very beneficial. For instance we found that comparing real systems to nature allows grasping the global picture quickly, sometimes the view let us pointing out network or protocol parts that could be simplified as pointed out before in XP (eXtreme Programming) [12]. Another advantage found was the possibility to bring nature sideeffects back to our design and convert them in new features or easy simplifications to the network under design.

Several works [21][22] have used Brownian motion [16] to model Ad-hoc networks, we want to provide a mathematical tool for applying other physics' concepts in these networks. In this line we left a door open for simulating heterogeneous [15] networks by using different kind of particles in future and physics concepts like entropy [17] or temperature in mixtures of two gases.

We aim the application of this methodology to the Ad-Hoc networks will provide new advantages. The structure of the paper starts with an intuitive common language definition of the model, next some basic "particle" formal foundations are established. Finally, some examples are described and some conclusions are drawn.

Permission to make digital or hard copies of all or part of this work for personal or classroom use is granted without fee provided that copies are not made or distributed for profit or commercial advantage and that copies bear this notice and the full citation on the first page. To copy otherwise, to republish, to post on servers or to redistribute to lists, requires prior specific permission and/or a fee.

InterSense '06. Proceedings of the First International Conference on Integrated Internet Ad hoc and Sensor Networks, May 30-May 31 2006, Nice, France

(C)2006 ACM 1-59593-427-8/06/05 ...\$5.00 


\section{INTUITIVE DESCRIPTION OF THE IDEA}

\section{A. Rationale}

Regarding the core or heart of this idea let us remark that its origin is not started by a single idea but by several postulates that joined together gave birth to this proposal. Let us see the generating axioms here:

- Are the actors more important than the messages, really? Contrary to our common thinking of a node as having more information - seems more "intelligent" - than a message (or signal), we propose to treat messages as the key elements instead of nodes (and networks), not only for Programmable Active Networks but for viewing any network.

- Can an effect be the consequence of only-one cause? Let us think that statement as only partially-true and consider that actually all effects were consequence of joining two, or more, elemental-causes or traditional-causes. The causes are in our approach the coincidence in space-time of two or more traditionally thought "elemental"-causes.

If we look at nature believing in the previous postulates, we will be able to recognize these behaviors. With this we do not mean the postulates are absolutely true but just a consistent view of reality. Given the complexity of present networking systems, we looked at the most elemental and smallest systems in nature, and that led us to choosing particle physics [10],[9] as the reference to model complex behaviors of adhoc networks.

Having found advantages in the particle physics level paradigms we experimented further using bigger conceptual elements such as molecules in chemical reactions. The use of macroscopic objects of daily use seems a particularly easy way to represent networks within this new particles-view. Designers can model abstract ideas based on experiences to which they can relate, with particles such as bowling collisions or billiard balls.

\section{B. A simple message-node communication example}

Let first see the example in figure 1, a particle interaction, and try to relate it to a traditional network exchange. Consider the collision of an electron and a proton to produce a neutron and a photon in figure 1a. The interaction between two particles in this way can serve for modeling a node with two input messages, and the particles resulting of the collision correspond to the output messages $\left(\mathrm{S}_{3} \mathrm{~S}_{4}\right)$.

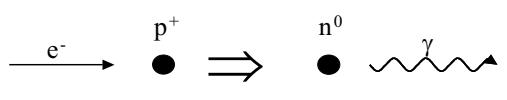

a)

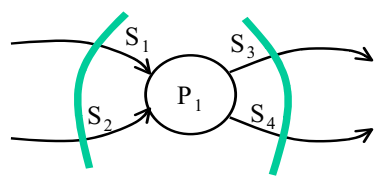

b)

Fig. 1 a) above, a representation of the collision $\mathrm{e}+\mathrm{p} \rightarrow \mathrm{n}+\gamma$; b) its view as packets $(\mathrm{S})$ and a network node or subnetwork $(\mathrm{P})$.
Intuitively we can see how the messages $S_{1} S_{2}$ in figurelb correspond to the electron and proton of figure 1a and the node $P_{1}$ is related to the point of its collision.

Collisions allow the introduction of space coordinates; and some of their advantages are making the model oriented to the topology of a network, as well as to graphical-views. It also allows a different view of networks: where messages are the real intelligent actors and routers simple colliding zones. However, the particle view is not limited to Active Networks and is usable for any network such as mobile and ad-hoc.

\section{Advantages of the particle dynamics representation}

This particle-representation could just be another way to model, simulate, manage or study networks. Let us mention now several benefits of the proposed approach, taking into account different points of view.

- Since the model associates zones, volumes, or space coordinates to nodes and sub-networks, it allows us to map the physical coordinates of a node. This is why we can say "it is topology oriented" and useful to model networks like position-based routing [7]. However, the designer may choose not to map modeling coordinates to a physical location, and use it as purely abstract information like in a concept map.

- In this representation, a sub-network located within a bigger subnet can be mapped to a collision volume, zone, or space area that is inside a bigger collision area. This makes the Particle-Dynamics particularly useful for modular and hierarchical representation of systems. For instance a network division in broadcast belts [2] can be mapped into different space coordinates for each layer.

- To finish with the abstract and space coordinates, we can identify zones producing particles as a special kind of source zones that can be mapped directly to interfaces with external networks. The input/output of a system can be represented straight forward as source/sink zones.

Second, we should consider that this model states that the importance of a system is in the messages more than in the traditional nodes or subnets. The application of this first postulate broke with the idea of intelligent nodes exchanging simple messages, and instead we have "intelligent" particles [our messages] being the real actors following "nature's rules" and colliding in simple zones [nodes]. Some advantages are:

- The representation methodology or model can ease and reduce the complexity of routers or network nodes and gives all the intelligence to the messages or packets. In this view the routers/ processes [zones] are simple areas or zones where the incoming messages [particles] could collide. It is not though for programmable-networks only, any network behavior can be described by particles and collisions (with the formalism of section III).

- If the particles are the main actors they have more power on the overall behavior of collision zones, and therefore as there are no main processors, a system can potentially modify itself becoming reconfigurable and an adaptable network. In this way the particle-dynamics methodology is aimed to be 
useful for describing adaptive networks for fault recovery [13], distributed systems [4], and security situations [8][11].

Other benefits and possibilities from others point of view:

- We can use Particles in traditional computational models, such as synchronous data-flow or finite state-machines, if we treat the messages as events and signals and the nodes as processes as described in the work of Lee-Sangiovanni [1].

- It is intended for different levels of abstraction, from the physical electric circuits definition of Network on Chip (NoC) to a higher level definition of behaviors where the "zones" represent other abstract concepts such as virtual networks.

- The visual and common experience view of this Particle representation can be used with elements such as billiard or snooker balls, if they were closer to the designer's everyday or common experience. That makes the modeling work more natural and user friendly.

Actually, the particle-dynamics approach applied to specifying and simulating networks can be managed by using a compiler or network design environment where we define a set of particles, collisions, and rules. Those are the basic elements to design and program with particles.

- A programming environment with well-known particles defined, and its rules already entered, can ease design tasks to designers familiar with daily physics such as billiards or bowling collisions.

- And also if we have a designing environment or compiler of particle-dynamics, it could have several flavors of network and protocols already preconfigured and allow the designers and implementations to choose among them.

This compiler or network design environment is not the main purpose of this work and will be addressed in future works. At present a basic prototype of particle-engine was the test with Wireless SIP (Session Initiation Protocol).

\section{FORMAL FOUNDATIONS OF THE APPROACH}

A robust formalization of the Particle-Dynamics model will show that it can be applied to a great number of network cases. A first way to validate the Particle-Dynamics is to demonstrate that it can be seen with analogous mathematics to the formal meta-model defined by Lee \& Sangiovanni [1] for processes and signals. This is a guarantee that the model is applicable to represent all network cases. We introduce and define formally here this proposed "Particle Dynamics" communication model.

\section{A. Defining Observed Particles [messages]}

A particle measurement or observed particle $p$ has a tag and a value; mathematically it is a member of the $\mathrm{T} \times \mathrm{V}$ set, where $\mathrm{T}$ and $\mathrm{V}$ are the sets of all possible tags and values respectively. A tag $t \in \mathrm{T}$ definition allows to model time, precedence relationships, but also other key properties like space-time in the Particle-Dynamics model. This is equivalent to what the computational reference framework [1] defines as an event. For representing networks each particle can represent a protocol message or packet.

\section{B. Defining Trajectory or Path [Conversations]}

A particle has space-time coordinates (tag) and features (values $v \in \mathrm{V}$ ) along its life history. This life trajectory or conversation $s$ can thus be defined as a subset of $\mathrm{T} \times \mathrm{V}$, a set of particles, or equivalently a member of the powerset $S \equiv$ $P(T \times V) \equiv e^{T \times V}$.

A trajectory or path is the set of representations of a particle at different times, i.e. just the space-time information portion contained in a signal. A trajectory corresponds to the $T(s) \subseteq T$ that was defined in the framework [1] for denoting the set of tags in a signal $s$.

\section{Defining Subnets and Space zones}

A tuple or vector $\boldsymbol{s}$ of $N$ paths is denoted as $b \equiv \boldsymbol{s} \in \mathrm{S}^{\mathrm{N}} S \times$ $S \times \ldots \times S$ and can represent the behavior of a node. A subnet or node $P$ was defined to be a subset of $S^{N}$ for some $N$, i.e. a set of behavior tuples $s$ or $b$.

We define a zone $z$ to be an index of any subnet (including the minimal subnet of just one node or router) in the set B of nodes $P_{1} P_{2} \ldots P_{M} \ldots$. The nodes can be indexed from 1 to $\mathrm{M}$ for instance, or with integers $-\infty$ to $+\infty$ and each of those indexes will be a zone $z / \exists \mathrm{P}_{\mathrm{z}}$.

Let us remember that among the set of all possible behaviors we could have as unique element the network representing the entire system, but it can also be split into a combination of smaller subnets. The modeler or designer can choose the subnets and nodes that better fit.

\section{Extending Tags}

The values in $\mathrm{V}$ can represent the operands and results of computation, in our model we see $\mathrm{V}$ as a multidimensional set for multiple particles' attributes. The set of events is seen in a generic way $E \equiv T \times V \equiv X_{1} \times X_{2} \times \ldots \times X_{D}$, where each $X_{i}$ is a set of coordinates in a given dimension numbered $i$. This matches the ordering and examples in [1], where some models used a bi-dimensional $w \times w$ set for $T$ and others suggest different types of values for $V$, here seen as more dimensions.

\section{E. The connections or link zones}

A composition of zones [equivalent to a composition of processes in the reference framework] - denotes the intersection of the behaviors of each of the zones, needing a careful selection of a common superset $\mathrm{S}^{\mathrm{N}+\mathrm{M}+\ldots}$ in order to intersect subsets of different sorts.

We define a link $\mathrm{C} \subset \mathrm{S}^{\mathrm{N}}$, equivalent to connection in [1], as a simple zone with two (or more) of the particle paths (signals/ messages) in the N-tuple are always identical. This serves to model the links among sensors, nodes, subnets and networks. In a link we can model flows of messages/ particles carrying the information exchange.

In Particle-Dynamics a big zone can have smaller zones and particle details inside it. As any network (or process) can be included in another network (process), a connection or link is a case of sub-zone (intersection) inside the bigger zone (set union) containing the corresponding nodes. A subnet can be included in other subnet, and we could use a double index to represent both super and sub-networks. 


\section{F. Future work}

Based on an abstract concept of collision, we can look at ways to particularize collisions rules: we can have concrete forms from deterministic rules to some uncertainty variable that could lead to statistical analysis.

The collisions could seem as a regular way to interconnect networks and nodes using the sequential messages in natural paths. But looking at Feynman[9] multiple-path representation of particles we realize that particle physics only knows data on detection/generation. Therefore it could be closer to reality to model using origin(source) and destination(target) zones only.

If we give some degree of uncertainty to the direction or time of a collision outcome we have a method for modeling systems with stochastic process and random[18][14] variables. For future works we started working with a special particle that collides with particles making them to "decay" with random times, and it served to model all random variables [24] in our developments to present.

For evaluation of systems we aim at extending the tags further with new dimensions that allow us to model other interesting concepts:

In this sense the particles' world can be seen as filled with fields and multi dimensional coordinates, if we give concrete roles to these dimensions we are able to obtain needed models. A new dimension called "object layer" allowed us modeling object oriented in experiments. The same dimension serves for modeling a "particle class" that could exploit the benefits of heterogeneity in ad-hoc networks [15].

When working with a large number of entities, the notions like entropy [17] borrowed from physics were already useful in existing works [23]. Now, based on this mathematical framework relating networks and physics, we can easily calculate other fields such as density or temperature. Works as [5] evaluated the average response time per node according to node velocity, showing a certain protocol was insensitive to nodes' velocity. In future works we could use physics terminology and talk of a protocol behavior depending (or not) of "nodes' temperature".

In wireless sensor networks a very important resource is energy. This particle model leaves a door open for adding a "Battery" dimension to observed particles, therefore it could be used to represent energy maps [19] showing the amount of energy available at each part of the network.

Performance evaluation requires adding "capacity" values to the links and nodes of a system. We plan to aply the particle model from specification to evaluation by using again these extensions to particles' tags. The capacity of a connection or link "zone" could be modeled with an extra dimension or with just closer space-time separation that makes messages to go through faster. Analogously, future works could add "power" or "cpu" dimensions at each zone for performance evaluation in future particle designs aimed for simulations and analysis. The formalism introduced in section III allows all these cases.

\section{REPRESENTING NETWORKS THROUGH THE PARTICLE DYNAMICS CONCEPTS}

The particles vision gives a high abstraction for concepts such as collision and classification of particles. From that vision we can generate several less-abstract models, depending on how we choose to detail a given part of the high abstraction.

For instance, looking at the way we observe particles the model allows us to define more details of the particle observation space-time. If we map observed time to an ordered tag set (as natural numbers) we have data-flow [3] or Kahn process networks. If the tags were real numbers timestamps we could define a discrete-event (DE) model.

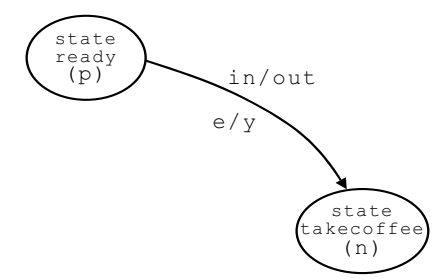

Fig. 2. State Machine classical representation.

The formalization in the reference [1] and in the previous section serves as a foundation for converting particles to other models, with formal works for many models [25] as Petri-nets or Finite State Machine (FSM). Since any FSM with states and transitions was demonstrated in [25][1] to be a subset of the meta-model[1], and particles were made equivalent to the meta-model in section III, we can use particles to model the FSM inside each node of an ad-hoc network. A basic particle FSM example is in figure 2 above.

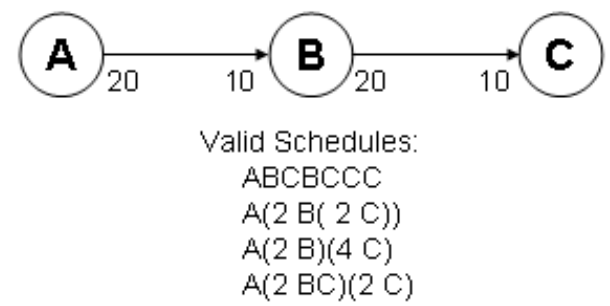

Fig.3. A reference SDF example, as in paper [3], can now be seen as three network nodes exchanging protocol messages.

\section{A. Particle-dynamic view of an SDF example model}

Let us recall [6][3] the Dataflow Process Networks (SDF), since they can be described as a case of the abstract metamodel [1] and the Particle-Dynamics is also applicable as an intermediate method of representation.

In figure 3, inspired in a dataflow process network, there is a directed graph with three nodes (network nodes, routers or sub-networks) and two arcs (representing sequences or streams of protocol messages or tokens).

To represent this in particle dynamics we will substitute first the nodes by zones. We will have zones $\mathrm{A}, \mathrm{B}$, and $\mathrm{C}$; 
where the $\mathrm{A}$ is an input link or source and $\mathrm{C}$ is an output. The interface zones allow relating the model to the real case they represent, but can also represent the physical interfaces that connect a node to the network.

Then we convert messages into particles, for instance each message from A to B can be modeled by a lambda particle $\Lambda^{0}$, or can also use a "composite" of elemental particles - as in nature several particles can tight together as one - is the case of proton-neutron nucleus or even groups of 2 protons and 2 neutrons $\left(\mathrm{He}^{4}\right)$ that we will use here. In the model of figure 4 we can assign the atoms $\mathrm{He}^{4}$ as produced in zone A for each input. Equally, each neutron or proton arriving to $\mathrm{C}$ is producing an output.

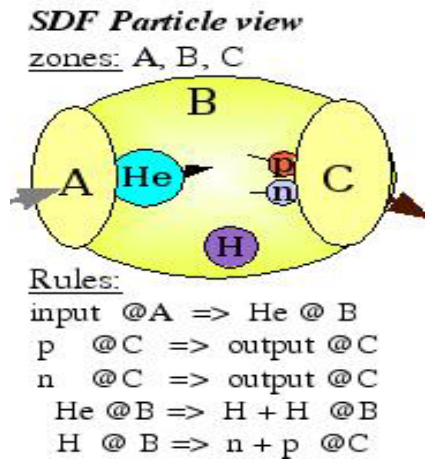

Fig.4. Particle view

There are two rules of collision / disintegration / or decay in zone $\mathrm{B}$, the $\mathrm{He}^{4}$ disintegrates into two $\mathrm{H}$ atoms. (The decay is actually formalized as a collision with a special particle that serves to control time and randomness). The second rule says that each $\mathrm{H}$ also decays and produces a neutron and proton. There is no physics knowledge needed for writing rules; in fact this was programmed thinking in Hydrogen when in physics this $\mathrm{H}$ would rather be a Deuteron atom $\mathrm{D}$; users write their own particles rules:

$$
\begin{aligned}
& H e-->H+H \\
& H-->n+p
\end{aligned}
$$

Like in a call-flow or sequential representation in particledynamics view we can have all the possible outputs. We can summarize that sequence corresponding to:

$$
\begin{aligned}
& \text { input --> He } \\
& \mathrm{He}-->\mathrm{H}+\mathrm{H} \\
& \mathrm{H}-->\mathrm{n}+\mathrm{p} \\
& \mathrm{H}->\mathrm{n}+\mathrm{p} \\
& \text { n --> outputs } \\
& \text { p --> outputs } \\
& \text { p --> outputs } \\
& \text { n --> outputs }
\end{aligned}
$$

The key for covering all message flows or possible schedules is that the particle rules do not define specific times of disintegration. This depends on concrete implementations of an SDF and can be decided later as described in [3].

\section{B. Complex Particles and heterogeneous systems}

Let us notice how in the last example we were thinking of using a complex elemental particle called lambda [20], but later chose to use particle compositions instead. This kind of particles and nature laws can be equally used and here is the previous example modeled with lambda decays.

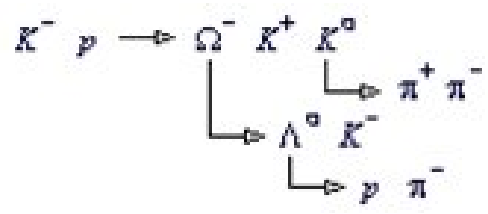

Fig.5 A set of particles fitting our example exist in nature, this real group of particle decays can be detected in large accelerators.

In this particular case we found a real set of particles that adjust to exactly the behavior we needed, as in figure 5 from observed collisions and data at CERN (European Organization for Nuclear Research). We can see "particle" as a generic term for objects that collide following the modelers' specific rules.

$$
\begin{array}{ll}
\Lambda^{0} \rightarrow n+\pi & \text { // possible: }\{\text { neutron or proton\} }+ \text { pion } \\
\pi \rightarrow \mu+v & \text { // decays into a muon and a neutrino } \\
n \rightarrow e+p & \text { // designers can add a decay like this one }
\end{array}
$$

More complex elements, such as groups of particles or even molecules in chemical reactions, can become a better model for some designers and network cases. In figure 4 the model based on bosons (n, p) and grouped-bosons $(\mathrm{H}, \mathrm{He})$ could have been designed equally using individual billiard balls and groups of balls tight together.

The formalism let the user to model networks formed by heterogeneous kind of nodes, sensors or routers; it is done by using different kind of particles where each particle type has a different behavior ( $\mathrm{H}$ decays differently than $\mathrm{He}$ ). We aiming at using those differences for the benefits described in [15] for heterogeneous sensor networks.

\section{EXPERIENCES AND TOOLS}

The particle-dynamics methodology was used in the specification and implementation of a set of software mobile SIP phones running in a special prototype ethernet network with a thousand different IP addresses in the lab.

The design was done graphically and the development effort suppoused a $30 \%$ saving on time respect to the plan as for the original development program.

When the software phones where running over a set of SUN servers we were able to achieve a performance a little over two hundred calls per second. This was the target of the traditional SIP prototype project, however it was achieved with a much simplier and shorter design effort than planned.

This methodoly and its benefits have passed in this way from a theoretical arena to the practical world. However the present state of the modeling or representation tool is still a prototype and this is a main disadvantage until a robust tool is put in service. The particle-engine or compiler is not the goal of this work, but rather having a formal base that could allow integrating existing tools from other models. Future efforts 
will aim to having an GNU project for specification, design, simulation, verification, and providing a network management environment consistent with the particle dynamics presented here and suported by a robust tool.

\section{CONCLUSIONS}

The particle-dynamics methodology is easily adapted to specify and analyze networks, especially those with mobile nodes and mobile sub-networks since each node is represented by a particle, that are naturally in movement.

We presented briefly the basis of the particle model with a mathematical formalization than fits into accepted formal models for representing computing systems. We provided an example of a particle modeling of a generic Kahn process network, and listed a set of advantages found using particles.

This application to deployment or simulation leads us to propose a new compiler or interpreter with a set of particle collision rules that will be the basis to design and study complex networks such as new Ad-Hoc or Sensor networks.

\section{REFERENCES}

[1] Edward A. Lee, and Alberto Sangiovanni-Vincentelli, . A Framework for Comparing Models of Computation, IEEE Trans. on ComputerAided Design Of Integrated Circuits And Systems, VOL. 17, NO. 12, DECEMBER 19981217

[2] Chlamtac, I.; Kutten, S.; On Broadcasting in Radio Networks--Problem Analysis and Protocol Design. IEEE Transactions on Communications, 1985, Volume 33, Issue 12, Dec 1985 Page(s):1240 - 1246

[3] Shuvra S. Bhattacharyya, Praveen K. Murthy, and Edward A. Lee Synthesis of Embedded Software from Synchronous Dataflow Specifications In Journal of VLSI Signal Processing Systems, Vol 21, No. 2, pages 151-166, June 1999. Kluwer Academic Publishers.

[4] Martinez C., GRIDs when the smaller outperform the big fish, ASTIC Journal, Vol 33 Mars 2005, Madrid, Spain

[5] Raverdy, P.-G.; Issarny, V.;2005 ; Context-aware service discovery in heterogeneous networks, World of Wireless Mobile and Multimedia Networks, 2005. WoWMoM 2005. Sixth IEEE International Symposium on a 13-16 June 2005 Page(s):478 - 480

[6] S. Edwards, Luciano Lavagno, Edward A. Lee, and A. SangiovanniVincentelli Design of Embedded Systems Formal Models, Validation, and Synthesis. Proceedings of IEEE, vol85, no3, mar1997, pp.366-390

[7] Stojmenovic, I., 2002, Position-based routing in ad hoc networks, ;Communications Magazine, IEEE Volume 40, Issue 7, July 2002 Page(s): 128 - 134

[8] Marvin V. Zelkowitz, Ioana Rus; 2001 ; Understanding IV \& V in a safety critical and complex evolutionary environment: the NASA space shuttle program, Publisher: IEEE Computer Society, Proceedings of the 23rd International Conference on Software Engineering, July 2001

[9] C. Elliott, D. Pearson, G. Troxel, 2003 Miscellany: Quantum cryptography in practice; Proceedings of the conference on Applications, technologies, architectures, and protocols for computer communications, August 2003 , Publisher: ACM Press

[10] R. Albert, A-L. Barabási, Statistical mechanics of complex networks, Reviews of Modern Physics 74, 47 (2002) Arxiv:cond-mat/0106096

[11] F. Damian, C. Martinez, "Internet Network security a pending matter", Proc. International Y Conference UNIV 1993, Rome Italy, April 1993

[12] Ahmed, A.; Fraz, M.M.; Zahid, F.A.;Some results of experimentation with extreme programming paradigm, 7th International Multi Topic Conference, 2003. INMIC 2003. 8-9 Dec. 2003 Page(s):387 - 390

[13] Weifeng X.; Ramanarayanan, R.; Tessier, R.; Adaptive fault recovery for networked reconfigurable systems; 11th Annual IEEE Symposium on Field-Programmable Custom Computing Machines, 2003. FCCM 2003. 9-11 April 2003 Page(s):143 - 152
[14] Calvo M., Martinez C, Alba J., "Simulacion VSAT". Satellite Aloha Protocol with BoNES Simulation. VIII Simposium Nacional URSI, pp 10-14. Valencia, Sept.1993

[15] M. Yarvis, N. Kushalnagar, H. Singh, A. Rangarajan, Y. Liu, S. Singh, "Exploiting Heterogeneity in Sensor Networks", IEEE International Conference INFOCOM 2005

[16] A. Einstein, Investigations on the Theory of Brownian Movement, ed. R. Fürth, translated by A.D. Cowper (1926, reprinted NY 1956); Einstein, Collected Papers, vol. 2, 170-82, 206-22.

[17] Gacs, P.; The Boltzmann entropy and randomness tests. Workshop on Physics and Computation,. PhysComp 17-20. 1994 Page(s):209 - 216

[18] G.D Hachtel, E. Macii,; A. Pardo, F. Somenzi, "Markovian analysis of large finite state machines", IEEE Transactions on Computer-Aided Design of Integrated Circuits \& Systems, Vol15 I12, 1996 Pp:1479-93

[19] Machado, M.; Goussevskaia, O.; Mini, R.; Rezende, C.; Loureiro, A.; Mateus, G.; Nogueira, J.; Data dissemination using the energy map, 2nd Annual Conference on Wireless On-demand Network Systems and Services, WONS 2005. Pp:139 - 148

[20] S. Eidelman et al (61 authors) Review of Particle Physics, PHYS LETT B 592 (1-4): 1-1109 Jul 15 2004,

[21] T. Camp, J. Boleng, V. Davies, A survey of Mobility Models in Ad Hoc Network Research, Wireless Communication \& Mobile Computing (WCMC) vol2 no5 pp. 483-502, 2002

[22] D. Blough, G. Resta, P. Santi, A Statistical Analysis of the Long-Run Node Spatial Distribution in Mobile Ad Hoc Networks, MSWiM Atlanta Georgia 2002

[23] Parunak H.V.D. \& Brueckner S.A.. "Entropy and Self-Organization in Multi-Agent Systems." In Proccedings of the Fifth International Conf. on Autonomous Agents (Agents' 2001), Montreal, Canada, 2001.

[24] Martinez, C; Estebanez, L. ; "Formalized methodology to treat NatureInspired Systems mathematically based on particle-dynamics physics", Proc. Adaptation in Artificial \& Biological Systems AISB 2006, Bristol

[25] S. Edwards, L Lavagno, E. A. Lee, A. Sangiovanni-Vincentelli, Design of Embedded Systems Formal Models, Validation, and Synthesis. Proceedings of IEEE, vol85, no3, mar1997, pp.366-390 